Claremont Colleges

Scholarship@ Claremont

All HMC Faculty Publications and Research

HMC Faculty Scholarship

7-1-1994

\title{
Subpicosecond Thomson Scattering Measurements of Optically Ionized Helium Plasmas
}

\section{T. E. Glover}

University of California - Berkeley

Thomas D. Donnelly

Harvey Mudd College

E. A. Lipman

University of California - Berkeley

A. Sullivan

University of California - Berkeley

R.W. Falcone

University of California - Berkeley

\section{Recommended Citation}

T.E. Glover, T.D. Donnelly, E.A. Lipman, A. Sullivan, R.W. Falcone, "Subpicosecond Thomson Scattering Measurements of Optically Ionized Helium Plasmas," Phys. Rev. Lett. 73, 78 (1994). doi: 10.1103/PhysRevLett.73.78

This Article is brought to you for free and open access by the HMC Faculty Scholarship at Scholarship @ Claremont. It has been accepted for inclusion in All HMC Faculty Publications and Research by an authorized administrator of Scholarship @ Claremont. For more information, please contact scholarship@cuc.claremont.edu. 


\title{
Subpicosecond Thomson Scattering Measurements of Optically Ionized Helium Plasmas
}

\author{
T. E. Glover, T. D. Donnelly, E. A. Lipman, A. Sullivan, and R. W. Falcone \\ Department of Physics, University of California at Berkeley, Berkeley, California 94720
}

(Received 2 February 1994)

\begin{abstract}
We present the first subpicosecond time-resolved temperature measurements of plasmas produced by high-intensity optical ionization. Thomson scattering is used to measure electron and ion temperatures of helium plasmas created by $125 \mathrm{fs}, 800 \mathrm{~nm}$ laser pulses focused to an intensity of $2 \times 10^{17} \mathrm{~W} / \mathrm{cm}^{2}$. We find that the electron temperature is accurately predicted by a tunneling ionization model. The measured ion temperature is consistent with direct heating by the laser pulse.
\end{abstract}

PACS numbers: $52.40 . \mathrm{Nk}, 52.25 . \mathrm{Rv}, 52.50 . \mathrm{Jm}$

Several authors have recently suggested that $\mathrm{x}$-ray lasers pumped by electron-ion recombination are feasible if cold electrons can be produced from the interaction of a high-intensity laser pulse with a gas sample [1]. Burnett and Corkum [2] used a model based on tunneling ionization and the resulting drift energy to predict that relatively cold electrons can be produced from such an interaction. Initial experimental tests of this prediction produced conflicting results. Corkum, Burnett, and Brunel [3] concluded that electron energies are reasonably well predicted by the tunneling model, while Mohideen et al. [4] concluded that the tunneling model significantly underestimates average electron energies. Both of these experiments were performed at low gas density $\left(<10^{11} / \mathrm{cm}^{3}\right)$. Recombination lasers, however, require relatively high gas density $\left(>10^{18} / \mathrm{cm}^{3}\right)$ which may result in additional heating mechanisms [5]. Experiments performed at high density $[6,7]$ have generally utilized longer laser pulses than optimal for transient recombination lasers. For example, the measurements of Offenberger et al. [6] were performed using 12 ps pulses; inverse bremsstrahlung (IB) dominated the electron heating and thermal conduction significantly modified the measured temperatures. The accuracy of the tunneling model, therefore, could not be assessed by these experiments.

In this work we use Thomson scattering to determine electron and ion temperatures of moderately high-density helium plasmas. We use a two-pulse technique to create and then probe the plasma on a time scale short enough that no significant plasma cooling occurs. Our experiments, performed in a regime where ionization heating determines electron energies, test the accuracy of the tunneling model at densities and on time scales relevant to recombination lasers.

The experimental apparatus is shown in Fig. 1. An initial laser pulse ( $125 \mathrm{fs}, 30 \mathrm{~mJ}, 800 \mathrm{~nm})$ ionizes helium gas and a second, colinear pulse at $400 \mathrm{~nm}$ probes the preformed plasma. The probe pulse is produced by frequency doubling the $800 \mathrm{~nm}$ pulse in a $1 \mathrm{~mm}$ KDP crystal. We calculate that the wavelength dependent index of refraction in KDP results in a maximum pump-probe delay of $80 \mathrm{fs}$. We vary this delay up to 1 ps by passing both pulses through a glass window. This two-pulse technique avoids complications that could arise from modification of the laser spectrum due to ionization induced blueshifting [8]. Such modifications could complicate interpretation of the Thomson-scattered spectrum and are minimized in these experiments because, at focus, our probe pulse sees a fully ionized $\mathrm{He}^{2+}$ plasma. As expected, measurements of the transmitted probe pulse spectrum revealed no spectral modification. Our peak ionizing laser intensity $\left(2 \times 10^{17} \mathrm{~W} / \mathrm{cm}^{2}\right)$ is a factor of 20 in excess of the intensity necessary [9] to produce $\mathrm{He}^{2+}$, which insures that $\mathrm{He}$ is fully ionized over the spatial dimension of our focused probe pulse. The pump and probe pulses are focused to spot sizes of $6.5 \mu \mathrm{m} \times 5 \mu \mathrm{m} \times 5 \mu \mathrm{m} \times 3 \mu \mathrm{m}$, respectively, using an $8-\mathrm{cm}$-focal-length off-axis parabolic mirror. We determined that the probe beam was contained within the focus of the ionizing beam by imaging the focal regions of both beams simultaneously using a charged coupled device camera. The peak intensity of the probe pulse was $2 \times 10^{16} \mathrm{~W} / \mathrm{cm}^{2}$.
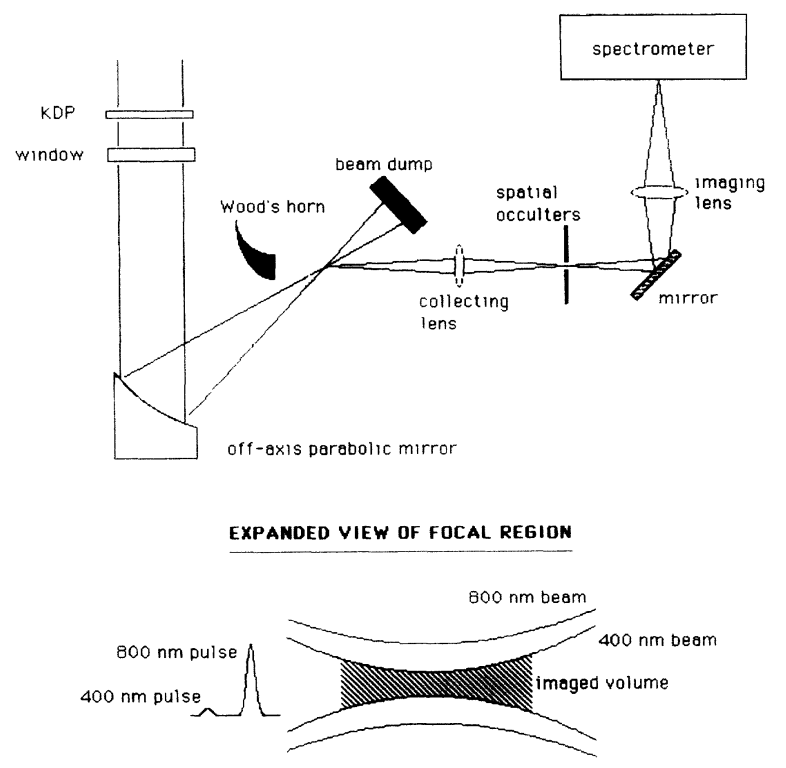

FIG. 1. Experimental layout. An initial laser pulse at $800 \mathrm{~nm}$ fully ionized $\mathrm{He}$ gas and a second pulse at $400 \mathrm{~nm}$ probed the resulting plasma. 
Thomson-scattered light was collected over a $f / 3$ collection angle using a $5 \times$ microscope objective which viewed a black surface (Wood's horn) behind the laser focus. The microscopic objective formed an image of the focus outside of the static-gas-filled target chamber; occulters at this image plane allowed us to discriminate against Thomson-scattered light originating from regions of incompletely ionized He. A second lens imaged the Thomson-scattered light through a crossed Czerny-Turner spectrometer $(1 / 8 \mathrm{~m}, 1200 \mathrm{~g} / \mathrm{mm}$ grating) followed by a gated microchannel plate intensifier (MCP) and optical multichannel analyzer. The gated MCP collected light for approximately $10 \mathrm{~ns}$; however, the effective time resolution for probing the plasma was the $125 \mathrm{fs}$ duration of our probe pulse. We gated the MCP to avoid interference from line emission in $\mathrm{He}$ ions; line emission dominated the detected light at late time. The level of stray-scattered light was typically less than $1 \%$ of the peak Thomsonscattered signal.

Thomson-scattered light was collected at an angle $(\theta)$ of $60^{\circ}$ with respect to the propagation vector $\left(k_{0}\right)$ of the 400 $\mathrm{nm}$ laser. The pump laser was polarized parallel to, and the probe laser was polarized perpendicular to, the scattering plane. Accordingly, we probed density fluctuations with wave number, $2 k_{0} \sin (\theta / 2) \cong k_{0}$. No statistically significant temperature differences were found between spectra taken at pump-probe delays of $1 \mathrm{ps}$ (glass window present) and $<80 \mathrm{fs}$ (no window); we therefore confirmed that plasma cooling was insignificant on the time scale of our experiments. Spectra were taken over a pressure range from 3 to 75 Torr. While data were also collected at higher gas pressures, no spectra corresponding to doubly ionized He could be obtained for pressures in excess of approximately 100 Torr. Experiments [10] indicate that for He gas pressures greater than approximately 100 Torr, ionization defocusing severely limits the achievable peak laser intensity so that $\mathrm{He}$ is no longer doubly ionized.

Spectra taken at gas densities of 50, 25, and 10 Torr are shown in Fig. 2. The central feature at $400 \mathrm{~nm}$ is called the "ion feature." The "electron features" (on either side of the ion feature) result from scattering due to electron density fluctuations associated with electron plasma waves modified by thermal motion. The data shown in Fig. 2 have been fitted by theoretical Thomson scattering spectra [11] convolved with both the spectral response of the spectrometer and the spectral distribution of the probe laser. The theoretical spectra assume Maxwellian electron distributions; we find good agreement with the experimental spectra at all pressures, as discussed below. (The measurements of Mohideen also indicate that the form of the initial electron energy distribution function produced by intense field ionization in $\mathrm{He}$ is well approximated by a Maxwellian distribution [4].) All fits are consistent with doubly ionized $\mathrm{He}$ at the specified density. Best fits are obtained for electron temperatures of nominally $40 \mathrm{eV}$; fits at the temperature extremes given by our error bars are seen in the inset to Fig. 2(b).
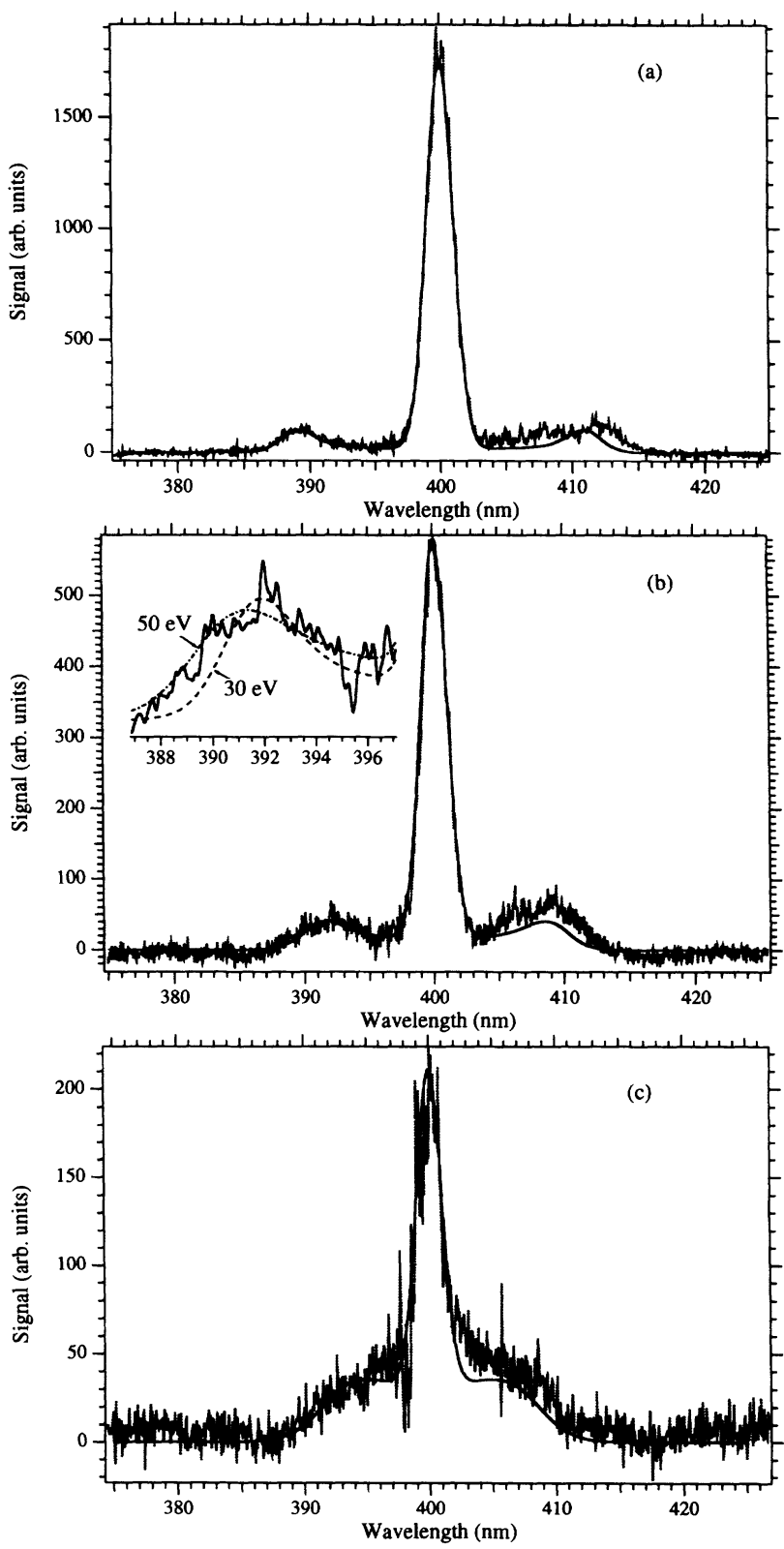

FIG. 2. Thomson spectra in helium (- - experimental data; - theory). (a) 50 Torr: fit to $40 \mathrm{eV}$ electron temperature and $3.6 \mathrm{eV}$ ion temperature. (b) 25 Torr: fit to $40 \mathrm{eV}$ electron temperature. Shown in the inset are fits for electron temperatures of 30 and $50 \mathrm{eV}$ (the ion temperature is $2.8 \mathrm{eV}$ ). (c) 10 Torr: fit to $50 \mathrm{eV}$ electron temperature and $2.5 \mathrm{eV}$ ion temperature.

While the fits to theory on the short wavelength side of the peaks in Fig. 2 are good, we note an asymmetry in the electron features and poor fits on the long wavelength side. We believe that this asymmetry is due to the presence of near-threshold stimulated Raman scattering (SRS). We find that we are able to significantly modify and/or enhance this long wavelength electron feature (in comparison to the short wavelength feature) by collecting light from different spatial regions of the plasma. 
The spatial dependence of this enhancement, along with the observation that the magnitude of the enhancement decreases with laser intensity, suggests that it is due to SRS. SRS has been observed to have strong spatial and laser-intensity dependencies [10]. The variability of the long wavelength electron feature renders it an unreliable diagnostic of electron temperature; electron temperatures are therefore determined from fits to the short wavelength electron feature. Experimentally measured temperatures were unaffected by the presence or absence of the observed SRS component.

We observe that reduction of the pump laser intensity by $50 \%$ did not change the electron temperature and this suggests that SRS did not significantly affect our temperatures. Wilks et al. [12] have investigated the influence of the SRS backscattering instability on the electron temperature for short-pulse laser-ionized plasmas; we calculate that SRS elevates the electron temperature by (at most) $10 \%$, so that we do not expect it to significantly affect the measured temperature. The observed invariance of measured temperatures to a $50 \%$ reduction in the pump laser intensity also suggest that ponderomotive heating of electrons [5] (which scales linearly with the laser intensity) is unimportant. Heating via ponderomotive expulsion of electrons is minimized in high-density plasmas due to the presence of large space charge fields that inhibit charge separation so that electrons do not move through spatial gradients of the focused laser spot [5].

The central feature at $400 \mathrm{~nm}$ in the spectra shown in Fig. 2 is the ion feature associated with electron density fluctuations caused by ions [11]. The spectral width of a short-pulse laser is large in comparison with the inherent width of this feature so that we are insensitive to the spectral form of the ion feature and instead sample its integrated power due to convolution with the laser spectrum. For low ion temperatures (high values of $Z T_{e} T_{i}$ ), the ion feature is a double peaked structure about the central laser frequency, and is due to electron density fluctuations associated with ion acoustic waves. For high ion temperatures (low $Z T_{e} T_{i}$ ), the ion feature is approximately Gaussian, centered at the central laser frequency, and reflects the ion thermal spread. Without resolution of the ion feature, as experimental spectrum taken at a single collection angle does not specify which of these ion scattering regimes dominates. This results from the fact that the intensity of the unresolved ion feature is a double valued function of ion temperature. The ion temperature (and subsequent specification of acoustic versus thermal scatter) can be uniquely determined from scattering spectra taken at different collection angles. A change in the collection angle changes the scale length of the fluctuations probed and therefore specifies the ion temperature. Spectra taken at collection angles of both $\theta=60^{\circ}$ and $120^{\circ}$ are consistent with fluctuations associated with acoustic waves. The resulting ion temperatures (see Fig. 3) range from $0.7 \mathrm{eV}$ at 3 Torr to $4.4 \mathrm{eV}$ at 75 Torr. Finally, we note that the intensity of the central ion feature was not a sensitive function of the spatial plasma region viewed or the presence or absence of an enhanced SRS component in the electron feature. These observations, along with the absence of a significant variation in the relative ion feature intensity with a $50 \%$ reduction in laser intensity, also suggest that stimulated Brillouin scattering did not significantly affect the ion feature.

The measured electron temperature as a function of gas density is also shown in Fig. 3. Both electron and ion temperatures were determined by finding best theoretical fits to several experimental spectra at each gas pressure and then taking the average of these fits. Error bars were determined by the range of temperatures that could reasonably fit the data. The electron temperatures are $40 \mathrm{eV}$ (3 Torr), $50 \mathrm{eV}$ (10 Torr), $40 \mathrm{eV}$ (25 Torr), $38 \mathrm{eV}$ (50 Torr), and $36 \mathrm{eV}$ (75 Torr). The nominal $10 \mathrm{eV}$ variation of the 10 Torr data from all other data is within the error of our measurement (typically 20\%). Within this error, we find that the electron temperature does not change over a 3-75 Torr pressure range.

As stated above, we calculate (using Ref. [13] that IB heating is unimportant for our experiments $(<2 \mathrm{eV}$ heating at 50 Torr). Ionization heating is computed using the quasistatic model of Corkum [3]. The probability of tunneling ionization is determined for each oscillation cycle of the laser pulse using the ionization rates of Ammosov [14]. The distribution of residual electron energies is given by the classical equations of motion for an electron in a laser field and equivalent temperatures are defined as two-thirds the predicted average energy. The predicted temperature for a $125 \mathrm{fs}$ pulse at $800 \mathrm{~nm}$ focused to $2 \times 10^{17} \mathrm{~W} / \mathrm{cm}^{2}$ is $40 \mathrm{eV}$, in good agreement with our measured temperatures.

In contrast to our results, the measurements of Mohideen [4] indicate that the tunneling model significantly underestimates electron energies. Those experiments yielded electron temperatures in $\mathrm{He}$ of $37 \mathrm{eV}$ for removal of the first electron and $140 \mathrm{eV}$ for removal of the second electron, implying an av-

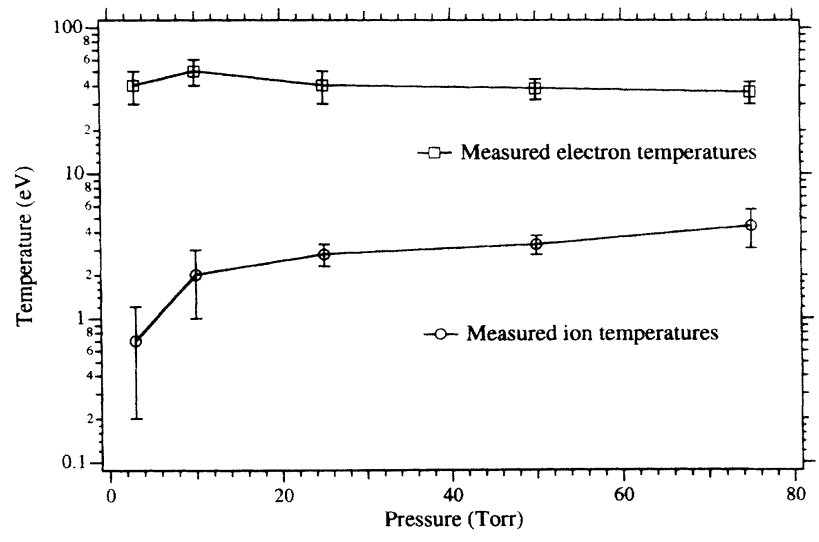

FIG. 3. Measured electron and ion temperatures as functions of gas pressure. 
erage, thermalized electron temperature in $\mathrm{He}^{2+}$ of $\approx 90 \mathrm{eV}$. For Mohideen's laser parameters $(820 \mathrm{~nm}$, $180 \mathrm{fs}, 2.5 \times 10^{16} \mathrm{~W} / \mathrm{cm}^{2}$ ), we calculate that the tunneling model predicts temperatures approximately a factor of 3 lower than those measured in his experiments. We considered the possibility that our experimental spectra preferentially reveal a colder $(37 \mathrm{eV})$ distribution while masking a hotter $(140 \mathrm{eV})$ distribution. Theoretical Thomson scattering spectra from these two electron distributions were summed; the resulting spectrum (at 50 Torr) indicates distinct peaks due to a $140 \mathrm{eV}$ distribution (at 413 and $387 \mathrm{~nm}$ ) which are a factor of 2 lower than the peaks due to a $37 \mathrm{eV}$ distribution (at 411 and $389 \mathrm{~nm}$ ). The peak due to the $140 \mathrm{eV}$ distribution would be observable (on the short wavelength side of the ion feature), but it is not present in the data.

While the origin of the discrepancy between our work and Ref. [4] is unknown, we note that time-resolved continuum slope measurements performed in our laboratory [15] support our conclusion that the electron temperature in $\mathrm{He}^{2+}$ is accurately predicted by the quasistatic model [3]. These continuum slope measurements were performed using $160 \mathrm{fs}, 616 \mathrm{~nm}$ laser pulses focused to an intensity of $2 \times 10^{16} \mathrm{~W} / \mathrm{cm}^{2}$. Using this technique, we measured a temperature of $22 \pm 4 \mathrm{eV}$ compared to the tunneling model prediction of $21 \mathrm{eV}$ for these laser parameters.

The magnitude of the ion temperatures, and their dependence on gas density, is consistent with a model of direct heating of ions by the laser pulse. Low ion kinetic energy leads to high (ion-ion) collisionality, so that ion-ion collisions dominate electron-ion collisions. We calculate ion heating as the product of the ion ponderomotive energy and the ion-ion collision frequency using the Spitzer [16] ion-ion collision frequency at an effective energy which is the sum of the ion thermal and ponderomotive energies. Ion heating saturates because the energy dependence of the collision frequency leads to a decrease in the heating rate for higher energy ions; predicted heating is therefore not strictly linear in gas density. The predicted ion temperatures are within the error of the measured data shown in Fig. 3. Our ion heating calculation is essentially an (ion-ion) inverse bremsstrahlung calculation; however, we note that in the dipole approximation inverse bremsstrahlung heating cannot result from collisions between particles with the same charge to mass ratio [17]. The agreement between our ion heating calculation and measured ion temperatures may be related to the presence of local electric field nonuniformities associated with space charge. The mechanism for ion heating requires further study.

In conclusion, we have used Thomson scattering to measure electron and ion temperatures in He plasmas produced by high intensity optical ionization. The measured ion temperatures increased with gas density and are consistent with calculations of direct heating by ion-ion collisions in the laser field. The measured electron temperatures did not vary significantly over a pressure range from
3 to 75 Torr; this result is consistent with our expectation that we are operating in a parameter space where electron temperatures are primarily determined by the optical ionization process. Contrary to the conclusions reached in Ref. [4], we find that the electron temperature is in good agreement with temperature predictions based on the tunneling ionization model. Our measurements suggest that electron energies can be accurately predicted by high field ionization theories and used to model potential recombination-pumped $\mathrm{x}$-ray laser systems.

The authors would like to thank Allan Offenberger, Hector Baldis, Wim Leemans, Umar Mohideen, David Villeneuve, William Kruer, and Scott Wilks for useful discussions and comments on our work, and Frank Patterson for loan of the OMA detector. T.E. G. would like to acknowledge support of an AT\&T CRFP fellowship. E. A. L. would like to acknowledge support of an NSF graduate fellowship. This work was supported by the U.S. Air Force Office of Scientific Research and through a collaboration with Lawrence Livermore National Laboratory under Contract No. W-7405-ENG-48.

[1] P. Amendt, D. C. Eder, and S. C. Wilks, Phys. Rev. Lett. 66, 2589 (1991); N. H. Burnett and G. D. Enright, IEEE J. Quantum Electron. 26, 1797 (1990); Y. Nagata et al., Phys. Rev. Lett. 71, 3774 (1993).

[2] N. H. Burnett and P. B. Corkum, J. Opt. Soc. Am. B 6, 1195 (1989).

[3] P. B. Corkum, N.H. Burnett, and F. Brunel, Phys. Rev. Lett. 62, 1259 (1989).

[4] U. Mohideen et al., Phys. Rev. Lett. 71, 509 (1993); U. Mohideen, Ph.D. thesis, Columbia University, 1993.

[5] B.M. Penetrante and J.N. Bardsley, Phys. Rev. A 43, 3100 (1991).

[6] A. A. Offenberger et al., Phys. Rev. Lett. 71, 3983 (1993). In more recent work, this group has observed Thomson scattering from a high-intensity 350 fs laser pulse used to ionize a plasma.

[7] W. P. Leemans et al., Phys. Rev. Lett. 68, 321 (1992).

[8] W. M. Wood, C. W. Siders, and M. C. Downer, Phys. Rev. Lett. 67, 3523 (1991).

[9] S. Augst et al., Phys. Rev. Lett. 63, 2212 (1989).

[10] J. Sheffield, Plasma Scattering of Electromagnetic Radiation (Academic, New York, 1975).

[11] A. Sullivan, Ph.D. thesis, University of California at Berkeley, 1993, available from University Microfilms Inc., 300 North Zeed Road, Ann Arbor, MI 48106-1346.

[12] S.C. Wilks (personal communication); S.C. Wilks et al. (to be published).

[13] R. D. Jones and K. Lee, Phys. Fluids 25, 2307 (1982).

[14] M. V. Ammosov, N. B. Delone, and V.P. Krainov, Sov. Phys. JETP 64, 1191 (1986).

[15] T.E. Glover, Ph.D. thesis, University of California at Berkeley, 1993, available from University Microfilms Inc., 300 North Zeed Road, Ann Arbor, MI 48106-1346.

[16] F.F. Chen, Introduction To Plasma Physics and Controlled Fusion (Plenum, New York, 1990).

[17] Y. Shima and H. Yatom, Phys. Rev. A 12, 2106 (1975). 